\title{
Editorial: Prioritising wellbeing through community and connection
}

\author{
Alicja Syska \\ University of Plymouth
}

This section of the Compendium opens with a powerful and moving reminder that the Covid-19 pandemic was not just a disruption, an inconvenience, and a challenge, but a devastating force that has had a profound impact on individual lives. Our students were often affected in unimaginable ways, including losing loved ones, losing their grounding and emotional resources, losing their confidence and social connections. Supporting students in the midst of all this loss, grief, withdrawal, and a sense of disenfranchisement in academia, required a lot of courage and a willingness to get hurt, but in the case of our authors, the impact of action and their willingness to give agency to students produced a beautiful event that validated grief, brought a sense of connection, and opened up hope.

This theme of uncertainty, anxiety, and disconnection, and the deep need to address it in an empathetic, kind, and supportive way permeates all the reflections. Authors of these pieces propose solutions such as adopting a pedagogy of kindness by connecting via audio and video messaging instead of email; co-constructing modules by listening to the student voice; creating compassionate spaces; and normalising learning challenges. Other initiatives demonstrate ingenuous attempts to make links between physical and mental health by encouraging walking for wellbeing, and efforts to acknowledge students' social needs by creating opportunities for networking and social interaction.

But for staff to be able to support students' wellbeing, they first had to look after themselves and one another. Here, educational developers' role was critical. In the reflections shared in this section, authors report on initiatives such as setting up a weekly Teachers' Lounge, creating targeted wellbeing programmes, and providing a virtual space for a 'free-wheeling' tea break designed to foster connection while not adding to the already very real burden of online meetings. Whether supporting staff or students, these 
authors propose thoughtful, caring responses to a crisis they were never trained to deal with and which stretched their own resources to the limit before they were able to find a productive rhythm in their work and life. 